\title{
Analysis of reactor-neutrino spectra fully based on gross theory of beta-decay emphasizing the special role of odd-odd FP nuclides
}

\author{
Tadashi Yoshida $^{1, \text { a }}$, Takahiro Tachibana ${ }^{2}$, and Satoshi Chiba ${ }^{1}$ \\ 1 Tokyo Institute of Technology, Laboratory for Advanced Nuclear Energy, Ookayama, Meguro-ku, Tokyo, Japan \\ 2 Waseda University, Advanced Research Institute for Science and Engineering, Okubo, Shinjuku-ku, Tokyo, Japan
}

\begin{abstract}
We applied the gross theory of $\beta$-decay to calculate the reactor antineutrino $\left(\bar{v}_{e}\right)$ spectra emitted from ${ }^{235,238} \mathrm{U}$ and ${ }^{239,241} \mathrm{Pu}$ samples under neutron irradiation by summing up all the contributions from a large number of decaying fission-products (FPs). Considering the special role of the odd $(Z)$-odd $(N)$ FPs in spectrum-shaping, we utilized the experimentally-known spin-parity of each odd-odd FP through the treatment proposed by Nakata, Tachibana and Yamada. Owing to this treatment, the consistency between calculated and experimental spectra was remarkably improved in a way expected from the nature of the gross theory of $\beta$-decay.
\end{abstract}

\section{Introduction}

Accurate and reliable knowledge of reactor-antineutrino energy spectra is indispensable in applications both for science and technology. One way to respond to this requirement is to convert the measured electron spectra from fissionable samples into the antineutrino spectra using the energy conservation of leptons in the $\beta$-decay process. Another is to compose these spectra by summing up all the contributions from a large number of decaying fission products (FPs).

Shreckenbach et al. measured the electron spectra for 4 uranium and plutonium isotopes and converted them into the antineutrino spectra [1-4]. Later, Mueller [5] and Huber [6] converted these electron spectra independently with their own refined methods.

The latter approach, the summation method, was adopted by Fallot et al. [7] and by Sonzogni et al. [8] making full use of the experimental decay data (decay schemes) of individual FPs accumulated up to the present time.

In the previous publication [9], the present authors applied the summation method fully based on the individual calculated spectra with the generation-2 version of the gross theory of beta decay [10-12]. Though the overall agreement of the calculated results with the experimental data by Shreckenbach et al. were fairly good, the $\mathrm{C} / \mathrm{E}$ values varied from 1.0 to 1.3 even in the $3 \sim 6 \mathrm{MeV}$ region where the gross approximation is most definitely valid and also the spectra are important for applications. In addition, large overestimation was found above $8 \mathrm{MeV}$ especially for the ${ }^{235} \mathrm{U}$ case. The calculation also indicated the dominance of the odd-odd nuclides in shaping the antineutrino spectra. From these points of view, we try to improve the prediction accuracy of the gross-theory-based summation calculation by introducing the Nakata-Tachibana-Yamada treatment[13] of the oddodd nuclides in the framework of the gross theory.

\section{Summation calculation of spectra}

Reactor antineutrinos are emitted from an aggregation of $\beta$-decaying FP nuclides in a reactor core. The source of the antineutrinos whose energy spectra will be analyze here is also the aggregation of $\beta$-decaying FPs in samples of various fissioning nuclides under neutron irradiation. Therefore, we have to start with the calculation of the concentration of individual FP nuclide making use of the coupled equations of burnup of the nuclear fuel for each decay-chain, namely,

$$
\begin{aligned}
\frac{d N_{i}(t)}{d t}= & -\left(\lambda_{i}+\sigma_{i} \phi\right) N_{i}(t)+\sum_{j} f_{j \rightarrow i} \lambda_{j} N_{j}(t) \\
& +\sum_{k} g_{k \rightarrow i} \sigma_{k} \phi N_{k}(t)+y_{i} F,
\end{aligned}
$$

where $N_{i}(t)$ is the concentration of $i$-th nuclide at time $t$. Symbols $\lambda_{i}, \sigma_{i}, \phi, f_{j \rightarrow i}, g_{k \rightarrow i}$ are the decay constant, the neutron capture cross-section, the neutron flux, and the branching ratios at decay and neutron-capture, respectively, all after the common notation. Further, $y_{i}$ and $F$ stand for the independent yield and the fission rate. A vast amount of actual values for these parameters were taken from JENDL FP Decay Data File 2011 and JENDL Fission Yield Data File 2011 [14] along with the structure of each $\beta$-decay chain. Hereafter we call these two files simply as JENDL. In the present calculation, the effect of the term including $\sigma$ is essentially negligible. By summing up all the contributions, we can calculate the antineutrino $\left(\bar{v}_{e}\right)$ spectra as

$$
I_{\bar{v}_{e}}\left(E_{\bar{\nu}_{e}}\right)=\sum_{i} N_{i} \lambda_{i} I_{i}^{\bar{\nu}_{e}}\left(E_{\bar{v}_{e}}\right) .
$$

\footnotetext{
a e-mail: tyoshida@nr.titech.ac.jp
} 


\begin{tabular}{|c|c|c|c|}
\hline \multirow{3}{*}{ 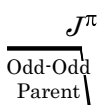 } & \multirow{2}{*}{$\begin{array}{c}J^{\pi} \\
\text { Spin-parity } \\
\text { of parent }\end{array}$} & \multicolumn{2}{|c|}{ Daughter nuclide } \\
\hline & & Ground state: $0^{+}$ & $1^{\text {st }}$ excited state: $2^{+}$ \\
\hline & $0^{+}$ & Fermi & --- \\
\hline & $0^{-}$ & $1^{\text {st }}$ forbidden rank 0 & $1^{\text {st }}$ forbidden rank 2 \\
\hline & $1^{+}$ & Gamow-Teller & Gamow-Teller \\
\hline & $1^{-}$ & $1^{\text {st }}$ forbidden rank 1 & $1^{\text {st }}$ forbidden rank 1,2 \\
\hline & $2^{+}$ & --- & Fermi \&Gamow-Teller \\
\hline & $2^{-}$ & $1^{\text {st }}$ forbidden rank 2 & $1^{\text {st }}$ forbidden rank $0,1,2$ \\
\hline & $3^{+}$ & --- & Gamow-Teller \\
\hline & $3^{-}$ & --- & $1^{\text {st }}$ forbidden rank 1,2 \\
\hline & $4^{-}$ & $\begin{array}{ll}-- & \\
-- & \end{array}$ & $1^{\text {st }}$ forbidden rank 2 \\
\hline & $4^{+}, 5^{+}, 5^{-}, \mathbf{6} \leq$ & --- & --- \\
\hline
\end{tabular}

Figure 1. Types of $\beta$-transitions from odd-odd nuclides to their daughters' low-lying states. (Transitions written in grey are ignored here considering their relative weakness.)

Here $N_{i}$ is the solution of Eq. (1) after a certain neutron-irradiation whose time duration is typical for each experiment $(10 \sim 50$ hours $)$ and $E_{\bar{v}_{e}}$ the kinetic energies of the antineutrino. The point is how to obtain the antineutrino spectra, $I_{i}^{\bar{\nu}_{e}}\left(E_{\bar{\nu}_{e}}\right)$, for each individual nuclide $i$. In order to calculate $I_{i}^{\bar{\nu}_{e}}\left(E_{\bar{\nu}_{e}}\right)$, we applied the gross theory of beta decay first developed by Takahashi, Koyama and Yamada [15-17] and then improved by Kondoh, Tachibana et al. [10-12]. We call this as GT2 after 'generation 2' of the gross theory. The details of these calculations related to Eq. (2) are given in the previous paper [9]. In our present calculation we included almost 900 unstable FPs with $I_{i}^{\bar{\nu}_{e}}\left(E_{\bar{v}_{e}}\right)$ calculated with GT2 mentioned above.

The gross theory is an average theory. It applies best to the quantities averaged over a large number of $\beta$-decaying nuclides. A typical example is the FP decay heat where the individuality of many contributing FPs cancels out each other in favor of the nature of the gross theory[18]. This will be an important point of the present paper.

\section{NTY treatment for odd-odd nuclides}

An important conclusion from the previous work [9] is that the odd-odd FP nuclides play a very important role in shaping the antineutrino spectra from fissioning materials. In reality, the odd-odd nuclides occupy more than $40 \%$ of all the contributors to the spectral value at $4 \mathrm{MeV}, 60 \%$ at $8 \mathrm{MeV}$ and almost $90 \%$ at $10 \mathrm{MeV}$. One reason for this is that the mass-difference between the odd-odd parent and its even-even daughter is very large because of the pairing effect of neutrons and protons. The gross theory, however, was not always good at predicting the half-lives of oddodd nuclides. In order to remedy this weakness, Nakata et al. [13] constructed a framework where they introduced the spin-parity of the odd-odd nuclides which decisively affect their decay properties beyond the average behavior. We describe this calculation as 'GT2 + NTY Calculation,' hereafter. It makes full use of the definite $0^{+}$and $2^{+}$ structure of the lowest levels of their even-even daughters. As a result, they confirmed that the NTY treatment is really effective to improve the prediction power of the half-lives of the odd-odd nuclides. As for distributed quantities such as the $\beta$-gtrength function or the $\beta$-ray energy spectrum, however, their method has not yet been validated up to now. The present study is the first test of the performance of the NTY treatment for a distributed quantiy deeply related to the odd-odd decay.

When we consider the $\beta$-transitions from an oddodd nucleus to the ground and the first excited states in the even-even daughter nucleus, the transition types are classified by using the selection rule of the spin and parity. These classifications of the $\beta$-transitions are shown in Fig. 1. For simplicity, we assume $0^{+}$and $2^{+}$for the ground state and the first excited state of the even-even nucleus, respectively, after the common structure of an even-even nucleus.

In the conventional GT2 model, all the transition types are included on an average. In the GT2 + NTY calculation, however, the transition type which satisfies the selection rule is enhanced, and all the other transitions are cut off. The enhancement factor here used is given as

$$
K(Z, N) \approx \frac{\left\langle 2 j_{n}+1\right\rangle\left\langle 2 j_{p}+1\right\rangle}{2 L+1} .
$$

Here $j_{n}$ is the angular momentum of the odd neutron or the odd neutron-hole, and $j_{p}$ is that of the odd proton or the odd proton-hole. Symbol $L$ stands for the rank of the corresponding $\beta$-decay operator. We assume that the $\beta$-transition occurs between the single-particle states with $j_{n}$ and $j_{p}$, and the rank of this $\beta$-decay operator is $L$. The bracket $<>$ means a local average of the value written inside the bracket. We calculated $j_{n}$ and $j_{p}$ by using a single-particle shell model. Equation (3) is obtained by considering the degeneracy of the $\beta$-decay strength calculated by the gross theory and the degeneracy of the actual $\beta$-decay strength. Since, in the gross theory, the oneparticle strength function is calculated so as to observe the sum rules for the one-particle transition, the sum rules of the whole nucleus are obeyed to some extent. Therefore, when we introduce the NTY-treatment, it should be noted that the enhancement or cut off the $\beta$-strength is done so as to obey the sum rules for the whole nucleus. In practice, the subtraction or addition of the strength is made at the lowest excited state still unmodified for compensation. In addition, the collective nature of the first excited $2^{+}$state in the daughter nucleus is taken into account by introducing a hindrance factor and the position of this state is assumed to be expressed by a function of mass number $A$.

\section{Results}

The upper panel of Fig. 2 shows the calculated results compared with the measured $\bar{v}_{e}$-spectrum (or more accurately, converted from the measured electron spectrum) for ${ }^{235} \mathrm{U}[1]$. The original GT2 calculation overestimates the experimental spectrum in the wide range of energy from 2 to $6 \mathrm{MeV}$ as a whole. Further in the region above $8 \mathrm{MeV}$, which is less important for applications but quite interesting from theoretical point of view, the overestimation is conspicuous. In order to remedy this, we introduced the NTY treatment for 175 odd-odd nuclides for which JENDL gives the definite spin-parities. First of all, the overestimation in the $2 \sim 6 \mathrm{MeV}$ region was well remedied. The improvement there realized by the NTY treatment is not, however, so demonstrative in the upper panel of Fig. 2 because of its logarithmic presentation. The ${ }^{238} \mathrm{U}$ case drawn in linear scale (the lower panel of Fig. 2) shows the improvement in a more explicit way. 

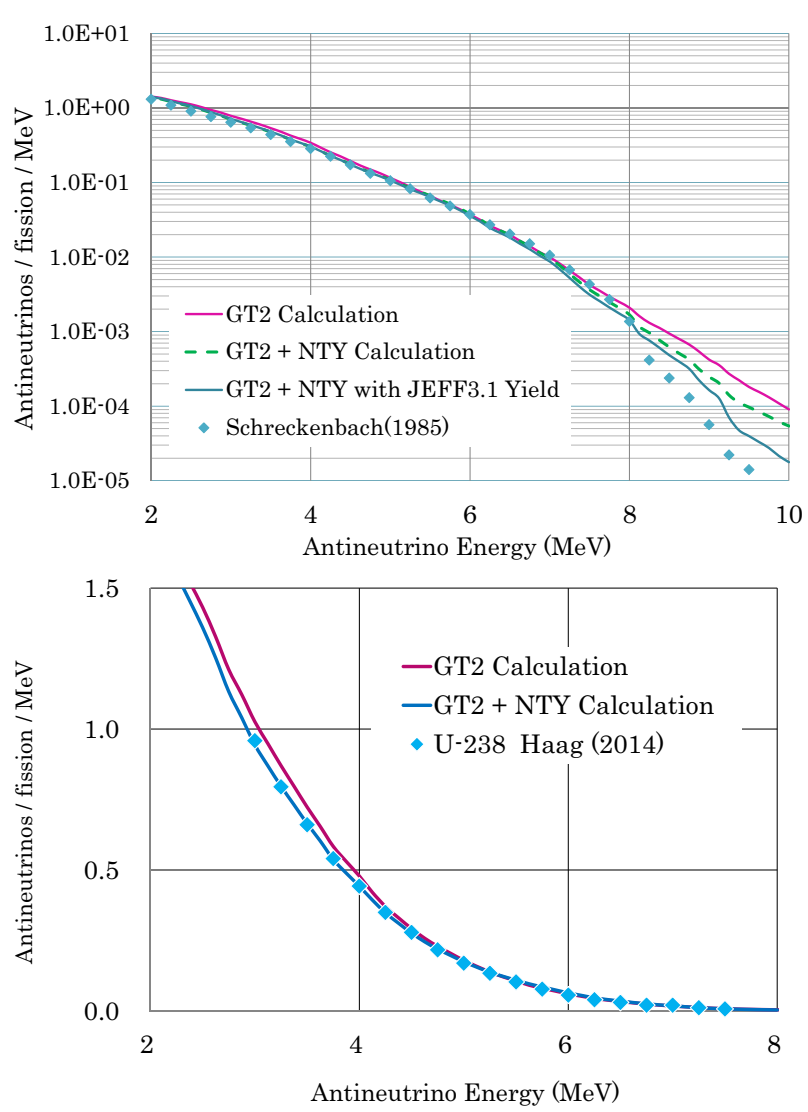

Figure 2. Effect of NTY treatment for odd-odd nuclides in the antineutrino spectrum of U-235 (top) and U-238 (bottom).

Table 1. Top 10 FPs contributing to ${ }^{235} \mathrm{U} \bar{\nu}_{e}$ spectrum at $9 \mathrm{MeV}$ (Definite value of spin-parity from JENDL, other information from the latest mass-chain evaluation in Nuclear Data Sheets).

\begin{tabular}{|c|c|c|c|}
\hline Nuclide & $\mathrm{Q}(\mathrm{MeV})$ & $\begin{array}{c}\text { Fractional } \\
\text { contribution }\end{array}$ & $\begin{array}{c}\text { Ground state } \\
\text { spin-parity }\end{array}$ \\
\hline $\mathrm{Rb}-94$ & 10.29 & 0.240 & $3^{-}$ \\
$\mathrm{Y}-98 \mathrm{~m}$ & 9.23 & 0.121 & 4 or 5 \\
As-86 & 11.54 & 0.087 & not given \\
Br-90 & 10.35 & 0.077 & not given \\
Y-100m & 9.46 & 0.075 & $5^{+}$ \\
Br-88 & 8.96 & 0.068 & $2^{-}$ \\
Rb-95 & 9.28 & 0.065 & - \\
As-84 & 10.09 & 0.042 & $3^{-}$ \\
As-88 & 12.60 & 0.038 & not given \\
Br-91 & 9.80 & 0.035 & - \\
\hline Sum & - & 0.848 & - \\
\hline
\end{tabular}

Then, we move to the higher energy region. Table 1 shows the top ten FPs contributing to the antineutrino spectral-value at $9 \mathrm{MeV}$. As far as this GT2 calculation is concerned, odd-odd nuclides dominate the high-energy part of the spectrum. As is seen here 4 nuclides are given their definite spin-parities among 8 odd-odds listed. These 4 FPs are responsible for the improvement seen in Fig. 2 (from red-solid to green-broken curve). If the spin-parity is well estimated for ${ }^{98 m} \mathrm{Y},{ }^{86} \mathrm{As},{ }^{90} \mathrm{Br}$ and ${ }^{88} \mathrm{As}$, it is expected that remaining gap can be filled. This invites us to a possibly fruitful trial of estimating the spin-parity of these rather exotic odd-odd nuclides, for example, based on the shell model.
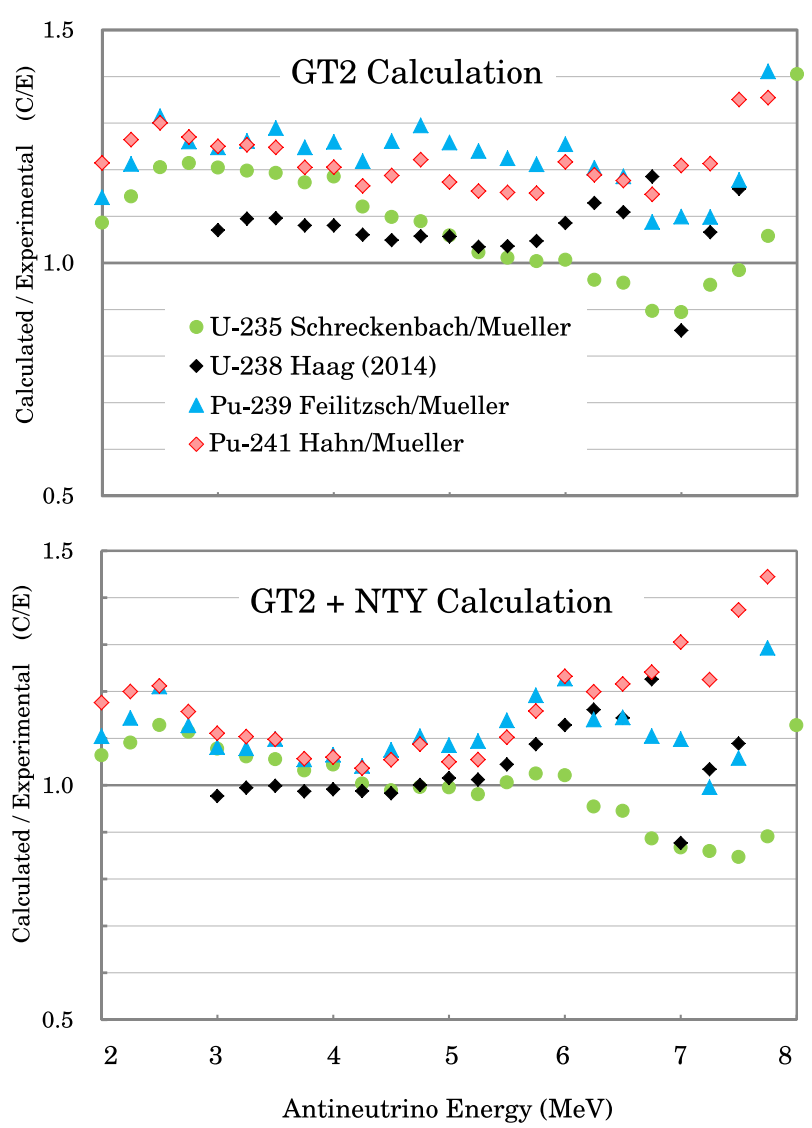

Figure 3. Calculated/Experimental values of antineutrino spectra before and after the application of NTY treatment for odd-odd nuclides.

In addition, the use of the JEFF3.1 fission yields [19] instead of JENDL further improves the consistency between the calculated and the experimental spectra above $8 \mathrm{MeV}$ (from green-broken to blue). The JENDL yields for ${ }^{235} \mathrm{U}$ seems to be suffered from some irregularities which cannot be justified easily. Quite recently a corrected version with the independent yield for ${ }^{86} \mathrm{Ge},{ }^{88} \mathrm{As}$ and ${ }^{100} \mathrm{Rb}$ being largely reduced was uploaded at the Web-site of JAEA Nuclear Data Center [20]. At $9.5 \mathrm{MeV}$ a half of the difference between the JENDL and JEFF3.1 can be filled with this reduction of the ${ }^{86} \mathrm{Ge}$ yield. For other three $\mathrm{U}$ and $\mathrm{Pu}$ isotopes, however, JEFF3.1 and JENDL yields give no essential difference as far as the antineutrino spectrum is concerned.

The summary of all the calculations here is given in Fig. 3. Here the $\mathrm{C} / \mathrm{E}$ (Calculated/Experimental) values are shown for all the fissioning nuclides studied here. As a whole the improvement realized by the NTY treatment is remarkable. In the medium energy range, where the canceling out of individualities of many nuclides works effectively in favour of the gross theory, the deviation of the $\mathrm{C} / \mathrm{E}$ value from 1.0 reduces by factors of $1 / 2 \sim 1 / 3$. Improvement cannot be seen in $6 \sim 8 \mathrm{MeV}$.

\section{Discussion}

Even after introducing the NTY treatment for the dominating odd-odd nuclides, the overestimation in the the energy region below $3 \mathrm{MeV}$ remains in a persistent way (Fig. 3). This requires a further consideration. First of all, there are two interesting types of contributions to 
this energy range among others. One is the low-energy antineutrinos emitted from low $Q_{\beta}$-valued FPs having long half-lives. Another is those emitted along with lowenergy transitions from rather high $Q_{\beta}$-valued parents to highly excited states in their daughters. For the former component, we have to accept it as it is because the the gross theory is not good, from its nature, at predicting the decay characteristics of low $Q_{\beta}$-valued nuclides. As for the latter component, this might suggest an overestimation of the high energy part of the $\beta$-strength function over the high $Q_{\beta}$-valued FPs in GT2. This possibility is expected to be checked by full use of the $\beta$-feeding data which is now being accumulated experimentally on the basis of the total gamma-ray absorption spectroscopy (TAGS) [21].

\section{Concluding remarks}

The authors applied the NTY treatment which takes into account the effect of the selection rules of $\beta$ transitions from an odd-odd parent to the low-lying states of its daughter in the framework of the gross theory. In comparison with the previous calculation based on simple GT2, the consistency between the calculation and the experimental data has remarkably improved. This result demonstrates the good applicability of the modern gross theory (GT2 + NTY) to aggregation of decaying FP nuclides which emit the high-energy antineutrinos. Among highly $Q_{\beta}$-valued FPs, however, there are many whose spin-parities are not yet determined experimentally. It is expected that reliable estimation of their spin-parities will further improve the prediction power of the theory.

\section{References}

[1] Schreckenbach, K., Colvin, G., Gelletly, W., Von Feilitzsch, F., Phys. Lett. 160B, 325-330 (1985)

[2] Von Feilitzsch, F., Hahn, A.A., Schreckenbach, K., Phys. Lett. 118B, 162-166 (1982)

[3] Hahn, A.A., Schreckenbach, K., Gelletly, W., Von Feilitzsch, F., Colvin, G., Krusche, B., Phys. Lett. B218, 365-368 (1989)
[4] Haag, N., Gütlein, A., Hofmann, L., Oberauer, L., Potzel, W., Schreckenbach, K., Phys. Rev. Lett. 112, 122501-1-5 (2014)

[5] Mueller, Th. A., et al., Phys. Rev. C 83, 054615 (2011)

[6] Huber, P., Phys. Rev. C 84, 024617 (2011)

[7] Fallot, M., et al., Phys. Rev. Lett. 109, 202504-1-5 (2012)

[8] Sonzogni, A.A., Johnson, T.D., McCutchan, E.A., Phys. Rev. C 91, 011301-1-5 (2015)

[9] Yoshida, T., Tachibana, T., Hagura, N., Chiba, S., Progr. Nucl. Energy 88, 175-182 (2016)

[10] Kondoh, T., Tachibana, T., Yamada, M., Progr. Theor. Phys. 74, 708-724 (1985)

[11] Tachibana, T., Yamada, M., Yoshida, Y., Progr. Theor. Phys. 84, 641-657 (1990)

[12] Tachibana, T., Yamada, M., Proc. Int. Conf. on exotic nuclei and atomic masses, Arles, 1995, eds. 84, 641657 (1990), eds. M. de Saint Simon and . Sorlin (Editions Frontueres, Gif-sur-Yvette, 1995) p. 763

[13] Nakata, H., Tachibana, T., Yamada, M., Nucl. Phys. A 74, 27-44 (1995)

[14] Katakura, J., JENDL FP Decay Data File 2011 and Fission Yield Data File 2011, Japan Atomic Energy Agency, Report JAEA-Data/Code 2011-25 (2011)

[15] Takahashi, K., Yamada, M., Progr. Theor. Phys. 41, 1470-1503 (1969)

[16] Koyama, S.I., Takahashi, K., Yamada, M., Progr. Theor. Phys. 44, 663-687 (1970).

[17] Takahashi, K., Progr. Theor. Phys. 45, 1466-1492 (1971)

[18] Yoshida, T., Nakasima, R., J. Nucl. Sci. Technol. 18, 393-407 (1981)

[19] Kelett, M.A., Bersillon, O, Mills, R.W., The JEFF3.1/-3.1.1 Radioactive Decay Data and Fission Yield Sub-Libraries, JEFF Report 20, NEA Report N.6287, Nuclear Energy Agency/OECD (2009)

[20] Katakura, J., Minato, F., Ohgama, K., EPJ Web of Conference 111, 08004 (2016)

[21] Algora, A., et al., Phys. Rev. Lett. 105, 202501 (2010) 